\title{
Stripline for Electromagnetic Susceptibility Testing - Input Impedance Matching
}

\author{
Zdeněk Kubík, Jiří Skála \\ Department of Applied Electronics and Telecommunications \\ Faculty of Electrical Engineering, University of West Bohemia \\ Pilsen, Czech Republic \\ zdekubik@kae.zcu.cz,skalaj@kae.zcu.cz
}

\begin{abstract}
This paper is focused on input impedance optimization of a stripline. The stripline for electromagnetic susceptibility testing represents a radiating structure which is composed of one active and one or more grounded conductors. The characteristic impedance of the stripline is given by the dimension and the material between conductors. If the characteristic impedance does not match to the output impedance of the RF amplifier, the impedance mismatch occurs and output circuits of the amplifier could be destroyed. The impedance matching circuit is necessary to be used in this situation. The impedance matching of the stripline using attenuators is shown in this paper; simulation results of $S$-parameters for different attenuator types were discussed.
\end{abstract}

Keywords-EMC; stripline; impedance matching; attenuator; electromagnetc susceptibility; simulation; VSWR; ISO 11452-5

\section{INTRODUCTION}

Today, all electronic devices are influenced by electromagnetic fields. The intensity of this interfering signal is dependent on the location of the device due to the distance from an interfering signal source. In some application, the devices could by exposed to the large intensity electromagnetic field - especially in the automotive industry. In order to avoid influencing of devices, there are a lot of EMC standards. All electronic devices placed on the market must meet the EMC standards.

Striplines are used for susceptibility testing of electronic devices to the electromagnetic field. The advantage of the striplines compared to the conventional method (a radiated field from a receiving antenna which is generated by an RF amplifier) is a relatively high intensity of the electromagnetic field. Striplines could be used for testing and measurement in a wide range of areas - in the automotive industry for vehicle components testing [1-4], consumers electronics (immunity testing of Sound and television broadcast receivers and associated equipment) [5, 6], integrated circuits immunity testing $[7,8]$ and radiated emission measurement [9] or gain measurement of the miniaturized antenna [10].

The impedance matching between the stripline and the high power RF amplifier is the most important part for a proper operation during the test - the unmatched stripline could cause the damage on the output stage of the amplifier. The impedance of the stripline depends on dimensions and material between conductors (air is considered for immunity testing). If the impedance does not meet the output impedance of the amplifier, an impedance matching unit should be used. Attenuators could be one of effective possibilities.

\section{STRIPLINE}

The characteristic impedance $Z$ of the stripline with the width of active conductor $W$ in meters and height $h$ which is the height of the active conductor above a ground plane in meters is [11]:

$$
\begin{gathered}
Z=\frac{60}{\sqrt{\varepsilon_{e}}} \ln \left(\frac{8 h}{W}+\frac{W}{4 h}\right)[\Omega] \quad \text { for } \frac{W}{h} \leq 1, \\
Z=\frac{120 \pi}{\sqrt{\varepsilon_{e}\left[\frac{W}{h}+1.393+0.677 \ln \left(\frac{W}{h}+1.444\right)\right]}}
\end{gathered}
$$

in Ohms for $\frac{W}{h} \geq 1$, where $\varepsilon_{e}$ represents an effective dielectric constant:

$$
\varepsilon_{e}=\frac{\varepsilon_{r}+1}{2}+\frac{\varepsilon_{r}-1}{2} \frac{1}{\sqrt{1+12 \frac{h}{W}}}
$$

The intensity of electric field between the stripline conductors is given by the equation [1]:

$$
|E|=\frac{\sqrt{P \times Z}}{h}[\mathrm{~V} / \mathrm{m}]
$$

where $P$ represents the input power to the stripline in watts, $Z$ characteristic impedance of the stripline in ohms and $h$ is the height of the active conductor above ground plane in meters.

\section{ATTENUATORS}

A passive attenuator represents a two port device; it is usually composed of resistive elements. The attenuator is connected between a source of the signal and a load (in our case the source represents a high power RF amplifier and the load represents the 
stripline) and reduce an output power by their attenuation. There are two basic connections of attenuator pad with the fixed attenuation, the first is the T pad and the second is the Pi pad attenuator.

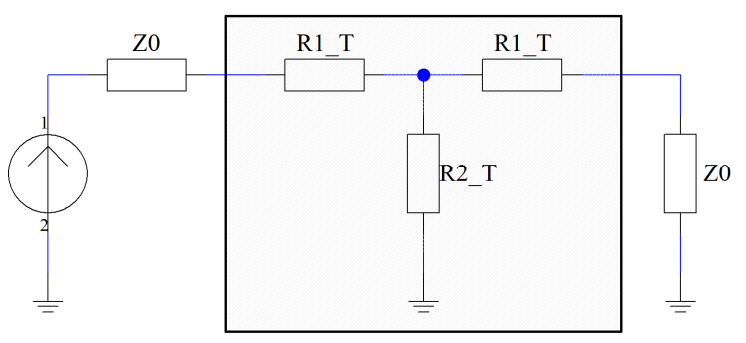

Figure 1. T pad attenuator connection

The connection of the $\mathrm{T}$ pad attenuator is shown in the box in Figure 1, where is placed the input voltage source with the impedance $\mathrm{Z} 0$ and the load with the same impedance Z0. Values of the resistor R1_T and R2_T are calculated by equations [12]:

$$
\begin{aligned}
& R_{-} T=Z 0\left[\frac{10^{\frac{A T T}{20}}-1}{10^{\frac{A T T}{20}}+1}\right] \quad[\Omega], \\
& R 2_{-} T=2 Z 0\left[\frac{10^{\frac{A T T}{20}}}{10^{\frac{A T T}{20}}-1}\right][\Omega],
\end{aligned}
$$

where ATT represents the value of the attenuation of the $\mathrm{T}$ pad attenuator.

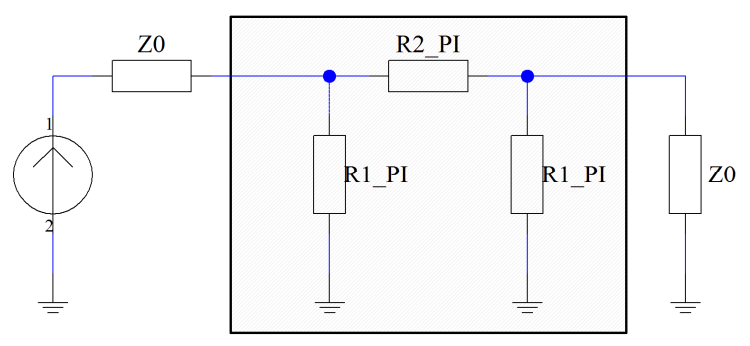

Figure 2. Pi pad attenuator connection

Figure 2 shows connection of the Pi pad attenuator, equations for resistors calculating are [12]:

$$
\begin{gathered}
R_{-} P I=Z 0\left[\frac{10^{\frac{A T T}{20}}+1}{10^{\frac{A T T}{20}}-1}\right][\Omega], \\
R 2_{-} P I=\frac{Z 0}{2}\left[10^{\frac{A T T}{20}}-\frac{1}{10^{\frac{A T T}{20}}}\right][\Omega] .
\end{gathered}
$$

\section{IMPEDANCE MATCHING}

The input parameters of the stripline could be described by the complex S-parameter S11. The VSWR (voltage standing wave ratio) is derived from S11:

$$
V S W R=\frac{1+\left|S_{11}\right|}{1-\left|S_{11}\right|}[-],
$$

where $\left|S_{11}\right|$ represents the magnitude of $S_{11}$ (also known as the reflection coefficient $\Gamma$ ).

The return loss $R L$ can be calculated by the equation:

$$
R L=-20 \cdot \log _{10}\left(\left|S_{11}\right|\right)[\mathrm{dB}] .
$$

If the stripline is connected directly to the $\mathrm{RF}$ amplifier (the ideal connection is considered) the reflected power can be calculated:

$$
P_{r e f}=P_{\text {out }}+R L[\mathrm{dBm}],
$$

where $P_{\text {out }}$ is the output power of the amplifier in $\mathrm{dBm}$.

Now the attenuator with the fixed attenuation ATT is connected between the stripline and the amplifier. The return loss of the system composed of the stripline and the attenuator is:

$$
R L_{A T T}=R L-2 \cdot A T T[\mathrm{~dB}],
$$

and a new value of $\left|S_{11}\right|$ with the attenuator is:

$$
\left|S_{11 A T T}\right|=10^{\left(\frac{R L_{A T T}}{20}\right)}[-] .
$$

The new value of VSWR with the attenuator:

$$
\operatorname{VSWR}_{A T T}=\frac{1+\left|S_{11 A T T}\right|}{1-\left|S_{11 A T T}\right|}[-],
$$

and a new reflected power of the system is:

$$
P_{\text {refATT }}=P_{\text {out }}+R L_{A T T}[\mathrm{dBm}] .
$$

From these equations - in term of the power amplifier - the impedance matching of the system with an attenuator is more beneficial than a system without the attenuator - there are lower values of the return loss as well as the reflected power. Of course, this solution has one negative part - the input power of the stripline is lower by the attenuation of the connected attenuator.

\section{ILLUSTRATIVE EXAMPLE}

The $50 \Omega$ stripline for susceptibility testing of automotive parts according to [1] was used for illustrative example in this paper. All simulations were performed in CST Microwave Studio 2015.

The dimensions of the $50 \Omega$ stripline are shown in Figure 3 . The active and the ground conductor were approximated by aluminum sheets with thickness 1 $\mathrm{mm}$.
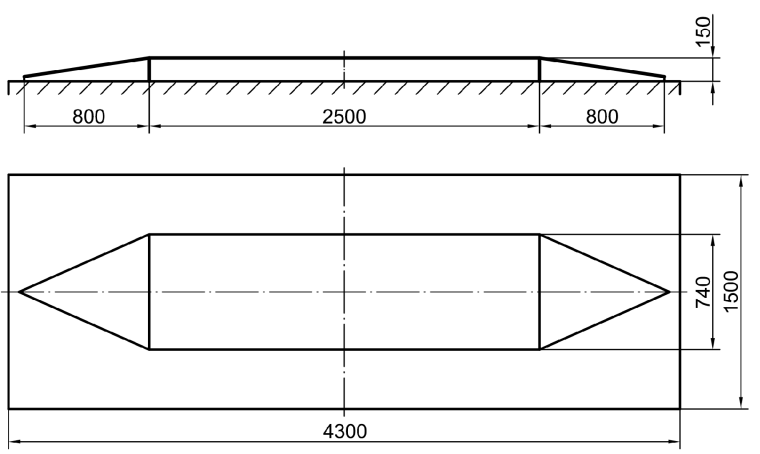

Figure 3. Dimensions of the stripline according to ISO 11452-5 
N-type connectors are used for stripline connections. Figure 4 represents a connection detail of a stripline feeder to the connector; four galvanized hex bolts were used for connectors mounting to the ground plane in the term of mechanical durability.

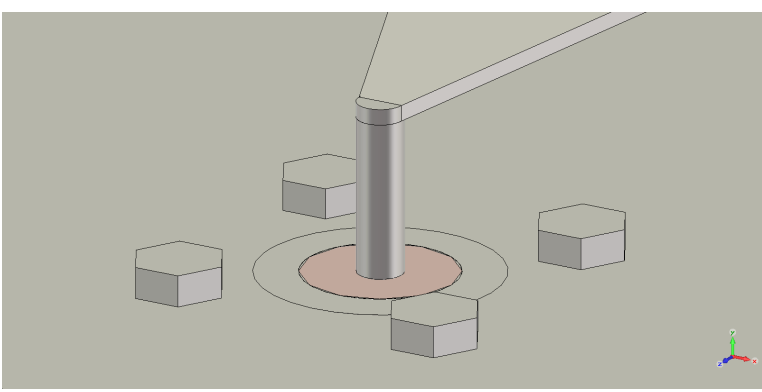

Figure 4. Connection detail of a stripline feeder to the N-type connector

At the first time, the impedance matching in the input connector S11 (S22 respectively) and the transmission between input and output connectors S21 (S12) were simulated. By the equation (9) the VSWR can be calculated.

Attenuators were connected between the input source and the stripline in the next steps and all Sparameters were recalculated.

Two types of attenuators were selected for simulations:

- $\mathrm{T}$ pad attenuator with attenuation $3 \mathrm{~dB}$,

- $\quad$ Pi pad attenuator with attenuation $6 \mathrm{~dB}$.

Parameters of used attenuators are shown in Table 1.

TABLE I. ATTENUATORS PARAMETERS

\begin{tabular}{|c|c|c|c|}
\hline Attenuator & $\mathrm{R} 1[\Omega]$ & $\mathrm{R} 2[\Omega]$ & Attenuation $[\mathrm{dB}]$ \\
\hline $\mathrm{T}$ & 8.55 & 142 & 3 \\
\hline PI & 150.48 & 37.35 & 6 \\
\hline
\end{tabular}

Here it should be noted that connection cables (between the amplifier and the attenuator and from the attenuator to the stripline) and both of attenuators which are composed of resistors were modeled as ideal, without any parasitic impedances.

Schematic diagram of the $\mathrm{T}$ pad attenuator for simulation is shown in Figure 5. Left hand side pad number 1 represents an external input port (approximation of RF amplifier output) with the fixed impedance $50 \Omega$. The second pad represents the second external port which substitutes the ideal $50 \Omega$ load.

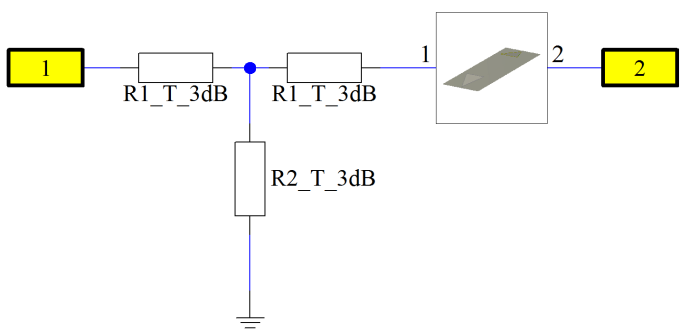

Figure 5. Schematic diagram of $\mathrm{T}$ pad attenuator for simulation
The symbol with a thumbnail of stripline represents $\mathrm{S}$-parameters simulation results from the $3 \mathrm{D}$ model.

Schematic diagram of the $\mathrm{Pi}$ pad attenuator for simulation is shown in Figure 6.

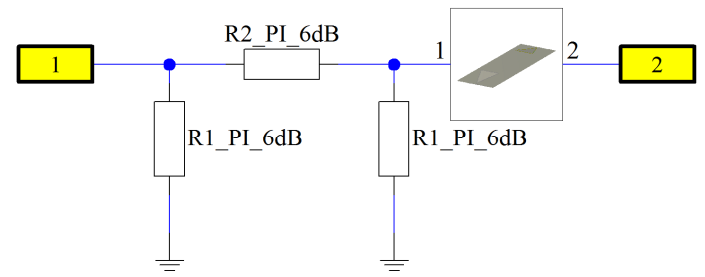

Figure 6. Schematic diagram of Pi pad attenuator for simulation

\section{SimUlation RESUlts}

Figure 7 shows results of S11 and S21 from 3D simulation of the $50 \Omega$ stripline.

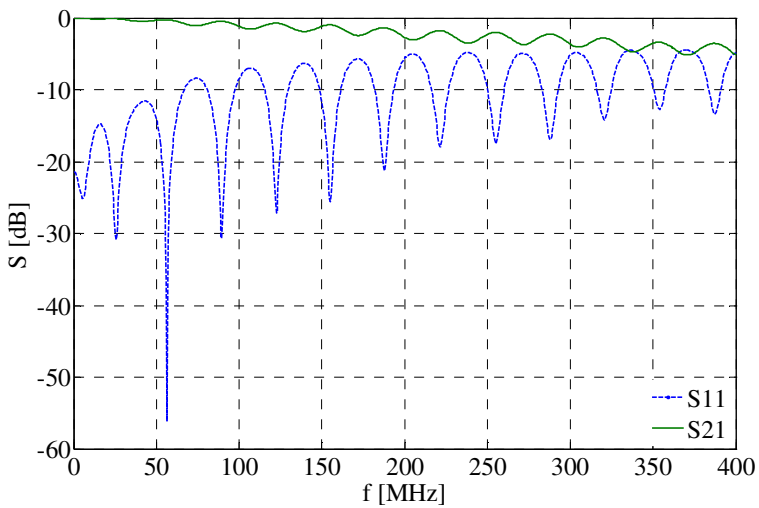

Figure 7. S11 and S22 dependence of the system without attenuator

Figure 8 represents dependencies of S11 parameter on the attenuation. The constant distance between two traces from simulation without the attenuator and simulation with the $3 \mathrm{~dB} \mathrm{~T}$ pad attenuator is $6 \mathrm{~dB}$, respectively $12 \mathrm{~dB}$ between traces from simulation without the attenuator and simulation with the $6 \mathrm{~dB} \mathrm{Pi}$ pad attenuator.

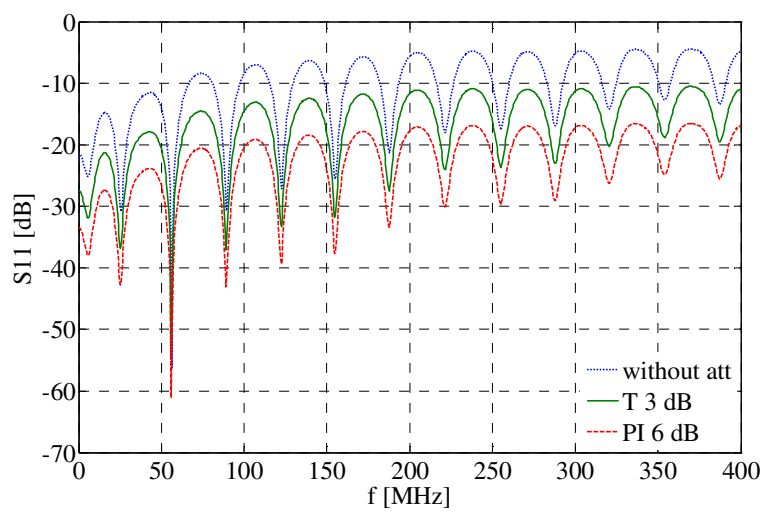

Figure 8. S11 dependence of the system on attenuation

The VSWR dependencies on attenuation are shown in Figure 9. For calculation was used the equation (9). The value of VSWR is crucial for the RF amplifier, values over 2:1 could threaten the output 
circuits of the amplifier. For example, the VSWR equals to 3.97 at the frequency $370 \mathrm{MHz}$ for connection without the attenuator, but VSWR $=1.84$ for connection with the $3 \mathrm{~dB}$ attenuator at the same frequency.

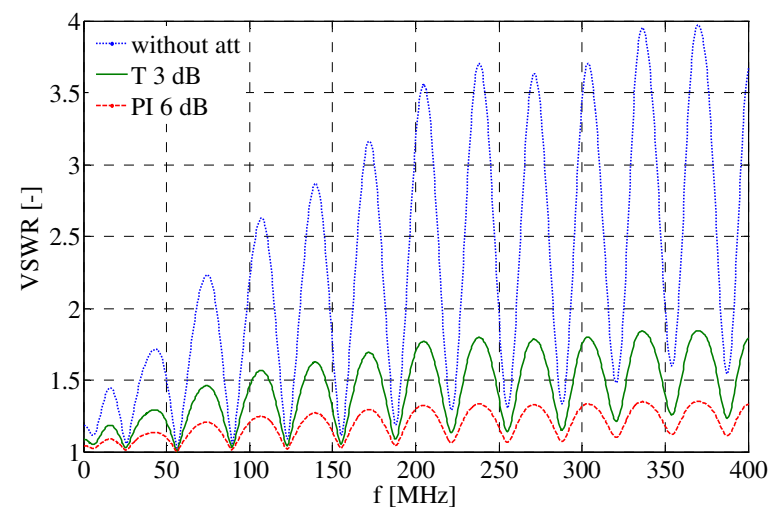

Figure 9. WSWR dependence of the system on attenuation

Figure 10 shows S21 dependencies on attenuation, there are visible estimated traces - with the constant distance $3 \mathrm{~dB}$ among them.

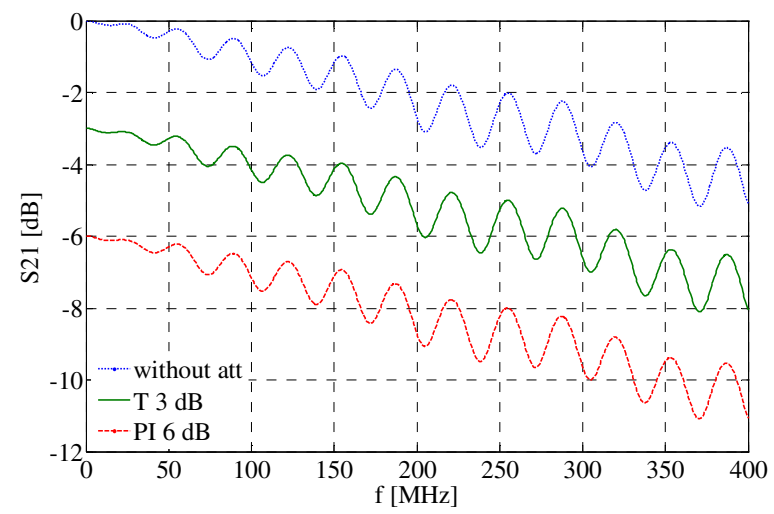

Figure 10. S21 dependence of the system on attenuation

\section{CONCLUSION}

This paper describes input impedance optimization of the stripline for electromagnetic susceptibility testing. The impedance could be adjusted by different ways - by dimensions of the stripline [13], by impedance matching circuits composed of resistors or transformers (baluns or ununs) [14], by attenuators. Impedance matching by using attenuators was shown in the paper.

The impedance matching is crucial for $\mathrm{RF}$ amplifiers, where the impedance mismatch between an output of the amplifier and the stripline could threaten the output stage of the amplifier; in the worst case, the output stage could be destroyed.

The attenuator improves the $\mathrm{S} 11$ parameters in $\mathrm{dB}$ of the stripline by doubling its attenuation. The improvement of S11 depends on the attenuation of the used attenuator. Of course, this method of impedance matching has one negative part - the output power from the RF amplifier is reduced by the attenuation of the attenuator at the stripline input.

\section{ACKNOWLEDGMENT}

This research has been supported by the project SGS-2015-002 and by the Ministry of Education, Youth and Sports of the Czech Republic under the RICE New Technologies and Concepts for Smart Industrial Systems, project No. LO1607.

\section{REFERENCES}

[1] ISO 11452-5:2002. Road vehicles - Component test methods for electrical disturbances from narrowband radiated electromagnetic energy — Part 5: Stripline. Second edition. 2002-04-15

[2] ISO 11452-1:2005. Road vehicles - Component test methods for electrical disturbances from narrowband radiated electromagnetic energy - Part 1: General principles and terminology. Third edition. 2005-02-01.

[3] M. Al-Hamid, R. Vick, M. Krüger and P. Wollmann, "Limitations of a stripline for immunity tests on road vehicle components," 2015 IEEE International Symposium on Electromagnetic Compatibility (EMC), Dresden, 2015, pp. 1232-1237.

[4] Yeon-Choon Chung, Tae-Won Kang and Dong-Chul Park, "Design and construction of stripline for measuring electromagnetic immunity of vehicular electrical cables," 1997 Proceedings of International Symposium on Electromagnetic Compatibility, Beijing, 1997, pp. 9-12.

[5] CISPR 20:2006. Sound and television broadcast receivers and associated equipment - Immunity characteristics - Limits and methods of measurement. Sixth edition. 2006-11-27.

[6] S. Hilavin and A. Kustepeli, "Design and Implementation of a TEM Stripline for EMC Testing," in IEEE Transactions on Electromagnetic Compatibility, vol. 56, no. 1, pp. 23-27, Feb. 2014.

[7] IEC 62132-8:2012. Integrated circuits - Measurement of electromagnetic immunity - Part 8: Measurement of radiated immunity - IC stripline method. First edition. 2012-07-06.

[8] J. Hwang, Y. Han, H. Park, W. Nah and S. Kim, "Radiated electromagnetic immunity analysis of VCO using IC stripline method," 2015 10th International Workshop on the Electromagnetic Compatibility of Integrated Circuits (EMC Compo), Edinburgh, 2015, pp. 147-151.

[9] IEC 61967-8:2011. Integrated circuits - Measurement of electromagnetic emissions - Part 8: Measurement of radiated emissions - IC stripline method. First edition. 2011-08-11.

[10] E. Kaverine, S. Palud, F. Colombel and M. Himdi, "A simple setup to measure the realized gain of miniaturized antennas in the HF band," 2016 10th European Conference on Antennas and Propagation (EuCAP), Davos, 2016, pp. 1-5.

[11] D.M. Pozar, "Microwave Engineering," fourth editon, Hoboken, NJ: Wiley, 2012. ISBN 978-0-470-63155-3.

[12] Van Valkenburg, M E. Reference Data for Engineers: Radio, Electronics, Computer, and Communications. 8th ed. Boston: Newnes, 1998. ISBN 0-7506-7064-9.

[13] M. Valek, T. Korinek and T. Bostik, "Design of Stripline for EMC Testing," 2008 14th Conference on Microwave Techniques, Prague, 2008, pp. 1-4.

[14] Franciscus Jozua van Dam, "A Stripline Antenna for Radiated Immunity Testing [online]," master thesis, University of Twente, EEMCS: Electrical Engineering, Mathematics and Computer Science, 2011, http://essay.utwente.nl/61201/1/ Stripline-Frank-20111027_14u45-v0073-Final_PDF3.pdf [Accessed 2017-03-02]. 